\title{
Regionalização e acesso à saúde nos estados brasileiros: condicionantes históricos e político-institucionais
}

\author{
Regionalization and access to healthcare in Brazilian states: \\ historical and political-institutional conditioning factors
}

Luciana Dias de Lima ${ }^{1}$

Ana Luiza d'Ávila Viana ${ }^{2}$

Cristiani Vieira Machado ${ }^{1}$

Mariana Vercesi de Albuquerque ${ }^{2}$

Roberta Gondim de Oliveira ${ }^{1}$

Fabíola Lana Iozzi ${ }^{2}$

João Henrique Gurtler Scatena ${ }^{3}$

Guilherme Arantes Mello ${ }^{4}$

Adelyne Maria Mendes Pereira ${ }^{1}$

Ana Paula Santana Coelho ${ }^{1}$

${ }^{1}$ Departamento de

Administração e

Planejamento em Saúde,

Escola Nacional de Saúde

Pública Sergio Arouca,

Fundação Oswaldo Cruz. R.

Leopoldo Bulhões 1480/

Prédio da ENSP/715,

Manguinhos. 21041-210

Rio de Janeiro RJ.

luciana@ensp.fiocruz.br

${ }^{2}$ Departamento de Medicina

Preventiva, Faculdade de

Medicina, Universidade de

São Paulo.

${ }^{3}$ Departamento de Saúde

Coletiva, Instituto de Saúde

Coletiva, Universidade

Federal do Mato Grosso.

${ }^{4}$ Departamento de Medicina

Preventiva, Universidade

Federal de São Paulo.

\begin{abstract}
This article examines the healthcare regionalization process in the Brazilian states in the period from 2007 to 2010, seeking to identify the conditions that favor or impede this process. Referential analysis of public policies and especially of historical institutionalism was used. Three dimensions sum up the conditioning factors of regionalization: context (historical-structural, political-institutional and conjunctural), directionality (ideology, object, actors, strategies and instruments) and regionalization features (institutionality and governance). The empirical research relied mainly on the analysis of official documents and interviews with key actors in 24 states. Distinct patterns of influence in the states were observed, with regionalization being marked by important gains in institutionality and governance in the period. Nevertheless, inherent difficulties of the contexts prejudice greater advances. There is a pressing need to broaden the territorial focus in government planning and to integrate sectorial policies for medium and long-term regional development in order to empower regionalization and to overcome obstacles to the access to healthcare services in Brazil.
\end{abstract}

Key words Regionalization, Health policy, Unified Health System, Healthcare planning, Access to healthcare services
Resumo O artigo aborda o processo de regionalização da saúde nos estados brasileiros no período de 2007 a 2010, com o objetivo de identificar as condições que favorecem ou dificultam esse processo. Utilizou-se o referencial de análise de politicas públicas e, particularmente, do institucionalismo histórico. Três dimensões sintetizam os condicionantes da regionalização: contexto (histórico-estrutural, político-institucional e conjuntural), direcionalidade (ideologia, objeto, atores, estratégias e instrumentos) e características da regionalização (institucionalidade e governança). A pesquisa empírica privilegiou a análise de documentos oficiais e entrevistas com atores-chave em 24 estados. Observaram-se combinações de fatores e padrões de influência distintos nos estados, sendo a regionalização marcada por importantes ganhos de institucionalidade e governança no período. Entretanto, dificuldades inerentes aos contextos comprometem maiores avanços. Há necessidade de ampliar o enfoque territorial no planejamento governamental e integrar políticas setoriais ao desenvolvimento regional de médio e longo prazo para fortalecer a regionalização e superar entraves ao acesso aos serviços de saúde no Brasil.

Palavras-chave Regionalização, Politica de saúde, Sistema Único de Saúde, Planejamento em saúde, Acesso a serviços de saúde 


\section{Introdução}

A descentralização é um fenômeno marcante das reformas dos Estados Nacionais desde o final dos anos $1970^{1}$. Essa agenda de reformas foi construída em um contexto de crise econômica mundial e redemocratização, e se expressa de maneira diversa nos sistemas de saúde, como forma para melhorar a eficiência, a efetividade e os instrumentos de accountability.

A experiência internacional sugere que, em países como Inglaterra, Alemanha, Itália, Espanha e Canadá, a descentralização articulou-se a processos de regionalização que possibilitaram a organização de redes de serviços, associadas à criação e fortalecimento de autoridades sanitárias regionais ${ }^{2}$. De modo geral, foram conferidas atribuições limitadas aos governos locais, enquanto os níveis regionais, vinculados aos entes estaduais ou provinciais nas federações, expandiram suas funções de planejamento e gestão sobre as políticas de saúde.

Já na América Latina, a descentralização e a regionalização foram conduzidas com graus variados de articulação, sempre com maior destaque para a descentralização, o que contribuiu para a fragmentação dos sistemas de saúde, com comprometimento do acesso e da continuidade da atenção ${ }^{3}$.

O descompasso entre esses processos também se verifica no Brasil. A descentralização do Sistema Único de Saúde (SUS) nos anos 1990 refletiu projetos econômicos e sociais de ideologias e finalidades distintas ${ }^{4}$, acoplando-se ao desenho federativo proposto na Constituição Federal de $1988^{5}$. As relações verticais foram priorizadas, tendo como foco a transferência de poder decisório, responsabilidades gestoras e recursos financeiros da União para os estados e, principalmente, os municípios. Mesmo considerando os movimentos de centralização legislativa e fiscal da função Estatal ${ }^{6-8}$, o período é testemunha da implantação de um modelo em que milhares de governos municipais adquiriram uma atuação abrangente no campo da saúde.

Porém, a fragilidade do planejamento regional das estratégias de descentralização do SUS comprometeu sua adequação às múltiplas realidades brasileiras. Com isso, não houve uma diversificação de políticas e investimentos que melhor relacionassem as necessidades de saúde às dinâmicas territoriais específicas visando à redução da iniquidade no acesso, na utilização e no gasto público em saúde?

Por sua vez, a regionalização ganha relevo no cenário nacional somente na virada dos anos 2000, quando da edição da Norma Operacional de Assistência à Saúde em 2001 (NOAS, reformulada em nova versão em 2002) $)^{10,11}$,e, mais recentemente, após a publicação do Pacto pela Saúde, em $2006^{12,13}$. Esse processo teve início tardio na maioria dos estados se conformando após sucessivos ciclos de descentralização ${ }^{14}$, induzidos e coordenados pela esfera federal ${ }^{15,16}$, sem uma revisão adequada do papel dos governos estaduais ${ }^{17}$.

Como consequência, os resultados da descentralização da saúde no Brasil são contraditórios e altamente dependentes das condições prévias locais. As características dos sistemas descentralizados de saúde refletem diferentes capacidades financeiras e organizacionais para a prestação da atenção à saúde e distintas disposições políticas de governadores e prefeitos ${ }^{18}$. Verificam-se problemas relativos à desintegração territorial de instituições, serviços e práticas, e dificuldades para a conformação de arranjos cooperativos entre os governos que garantam o acesso integral à saúde ${ }^{19}$.

$\mathrm{O}$ atraso na implantação de estratégias de regionalização no âmbito do SUS requer explicação. Contribuir para essa discussão é o principal propósito deste artigo, que tem como objeto os processos de regionalização da saúde em curso nos estados brasileiros no período de 2007 a 2010. Esse período corresponde ao momento de implantação do Pacto pela Saúde, cujas diretrizes visavam, entre outros, fortalecer a regionalização por meio da negociação e a pactuação intergovernamental ${ }^{20}$, com valorização das esferas estaduais e municipais, e a criação de novas instâncias de cogestão regional (os Colegiados de Gestão Regional) $)^{21}$.

Nesse estudo, compreende-se a regionalização como um processo técnico-político condicionado pela capacidade de oferta e financiamento da atenção à saúde, mas também pela distribuição de poder e pelas relações estabelecidas entre governos, organizações públicas e privadas e cidadãos, em diferentes espaços geográficos ${ }^{22}$.

Além disso, a regionalização envolve: o desenvolvimento de estratégias e instrumentos de planejamento, a coordenação, a regulação e o financiamento de uma rede de ações e de serviços de saúde no território ${ }^{23-25}$; a incorporação de elementos de diferenciação e diversidade socioespacial na formulação e implementação de políticas de saúde ${ }^{14}$; a integração de diversos campos da atenção à saúde e a articulação de políticas econômicas e sociais voltadas para o desenvolvimento e a redução das desigualdades regionais ${ }^{26}$.

Cabe destacar os significados associados às regiões para a organização político-territorial do 
SUS. As regiões, lócus principal dos processos de regionalização, constituem-se como base territorial para o planejamento de redes de atenção que possuem distintas densidades tecnológicas e capacidades de oferta de ações e serviços de saúde. São também espaços privilegiados de articulação intergovernamental para a condução do sistema de saúde.

A própria noção de rede regionalizada de atenção à saúde pressupõe a região como atributo fundamental para sua organização e funcionamento. Esta se constitui por um conjunto de unidades (ou pontos de atenção), de diferentes funções, complexidades e perfis de atendimento, que operam de forma ordenada e articulada no território, de modo a atender as necessidades e demandas da população ${ }^{25}$.

Por seus significados e pelas relações existentes entre regiões e redes de atenção, considera-se que o avanço do processo de regionalização tende a interferir positivamente no acesso à saúde, pois permite: observar os determinantes sociais de saúde no modo como estes se expressam no território; projetar necessidades de organização dos serviços de forma ampla incorporando diferentes campos da atenção e visão de futuro; atender uma população que não necessariamente se restringe aos territórios municipais; estabelecer portas de entrada e hierarquia tecnológica com base em parâmetros de necessidade e utilização dos recursos disponíveis; utilizar melhor os recursos humanos e tecnológicos presentes na região de forma a desbloquear fluxos e garantir resolutividade na atenção; disponibilizar recursos sociais e políticos que incentivem o compartilhamento de responsabilidades entre os governos nos sistemas de saúde.

Faz-se necessário identificar as condições que favorecem ou dificultam esse processo para a compreensão de possíveis entraves à ampliação do acesso à saúde no Brasil.

A importância readquirida pelos governos estaduais a partir dos anos $2000^{17}$, associada à diversidade territorial do país e à retomada do papel do Estado no desenvolvimento ${ }^{22}$, sugerem algumas questões para a investigação da regionalização: que fatores condicionantes desses processos explicam a diversidade de situações encontradas? Considerando a variedade de contextos, é possível a identificação de estágios similares da regionalização da saúde nos estados? Existem processos e mecanismos distintos que explicam estágios avançados de regionalização? Que razões limitam ou potencializam a regionalização da saúde nos estados?
Essas questões orientaram a pesquisa apresentada neste artigo, cujo principal objetivo foi analisar os condicionantes da regionalização da saúde tendo em vista a identificação das influências e dos padrões preponderantes nos estados brasileiros.

\section{Metodologia}

O trabalho ancorou-se no referencial de análise de políticas públicas ${ }^{27}$ e, particularmente, na abordagem do institucionalismo histórico ${ }^{28-30}$, tendo como pressuposto a influência das instituições nas estratégias e percepção dos atores, nos rumos, trajetórias e conteúdo das políticas.

Buscou-se identificar aspectos históricos, políticos, sociais e econômicos relacionados aos contextos dos diferentes estados brasileiros. Destacaram-se, ainda, as regras formais e informais que regem a atuação dos governos e que influenciam as escolhas políticas e os atores mobilizados na regionalização.

Três dimensões desdobradas em categorias sintetizam o referencial analítico dos condicionantes da regionalização nos estados (Quadro 1): contexto (histórico-estrutural, político-institucional e conjuntural), direcionalidade (ideologia, objeto, atores, estratégias e instrumentos), características da regionalização (institucionalidade, governança). Foram também verificados os impactos institucionais resultantes desse processo.

Essa abordagem assume que a regionalização é influenciada pelas características específicas da sua implementação na esfera estadual e, ainda, pelos rumos ou direção que se deseja imprimir ao processo. Os fatores que condicionam a regionalização nos estados também permitem explicar seus resultados, bem como seu avançado ou incipiente estágio de consecução.

A regionalização pode ser favorecida ou dificultada por um contexto histórico-estrutural (história da conformação do estado e de suas regiões, dinâmicas socioeconômicas e características geopolíticas dos estados), político-institucional (trajetória da política de saúde e da regionalização, modo de funcionamento das instituições e papel desempenhado pelas organizações envolvidas) e conjuntural (prioridade da regionalização na agenda dos governos, situação político-econômica do estado e perfil dos dirigentes da saúde) que varia entre os estados.

A direcionalidade expressa uma dada ideologia e a capacidade dos governos formularem uma orientação para o processo ${ }^{31}$, definindo os ato- 
Quadro 1. Referencial analítico da pesquisa.

\begin{tabular}{|c|c|c|c|}
\hline Dimensões & Categorias & Elementos principais & $\begin{array}{c}\text { Influência e padrões } \\
\text { predominantes }\end{array}$ \\
\hline \multirow{3}{*}{ Contexto } & $\begin{array}{l}\text { Histórico- } \\
\text { estrutural }\end{array}$ & $\begin{array}{l}\text { - Histórico de conformação do estado e de suas regiões } \\
\text { - Dinâmica socioeconômica e características geopolíticas } \\
\text { do estado }\end{array}$ & $\begin{array}{l}\text { - Favorável } \\
\text { - Desfavorável } \\
\text { - Indefinido }\end{array}$ \\
\hline & $\begin{array}{c}\text { Político- } \\
\text { institucional }\end{array}$ & $\begin{array}{l}\text { - Trajetória da política de saúde e da regionalização no estado } \\
\text { - Modo de funcionamento das instituições políticas e regras da } \\
\text { saúde } \\
\text { - Peso do privado na rede de serviços } \\
\text { - Papel desempenhado pelas organizações no sistema de saúde } \\
\text { (secretaria estadual e municipais, COSEMS, CIB) }\end{array}$ & $\begin{array}{l}\text { - Favorável } \\
\text { - Desfavorável } \\
\text { - Indefinido }\end{array}$ \\
\hline & Conjuntural & $\begin{array}{l}\text { - Prioridade da regionalização na agenda dos governos e da } \\
\text { Secretaria de Estado de Saúde } \\
\text { - Situação político-econômica do estado } \\
\text { - Perfil dos dirigentes da saúde }\end{array}$ & $\begin{array}{l}\text { - Favorável } \\
\text { - Desfavorável } \\
\text { - Indefinido }\end{array}$ \\
\hline \multirow[t]{5}{*}{ Direcionalidade } & Ideologia & $\begin{array}{l}\text { - Gerencial: foco nas mudanças organizacionais do Estado para } \\
\text { atuação na saúde } \\
\text { - Econômico-mercantil: mercantilização da provisão dos serviços } \\
\text { e estímulo à corresponsabilização privada no financiamento das } \\
\text { ações e serviços } \\
\text { - Democrática-participativa: ampliação da participação dos atores } \\
\text { envolvidos } \\
\text { - Equidade: melhoria das condições de saúde, ampliação do acesso } \\
\text { e integralidade } \\
\text { - Determinantes sociais: articulação de políticas voltadas para a } \\
\text { promoção da saúde } \\
\text { - Desenvolvimentismo: articulação de políticas sociais e } \\
\text { econômicas voltadas para o desenvolvimento e bem-estar }\end{array}$ & - Não se aplica \\
\hline & Objeto & $\begin{array}{l}\text { - Definição de região: delimitação territorial para organização do } \\
\text { sistema de saúde } \\
\text { - Organização de redes e fluxos: definição de polos, integração e } \\
\text { articulação de ações e serviços, sistema de referência e } \\
\text { contrarreferência e dos fluxos de encaminhamento } \\
\text { - Ampliação da capacidade instalada: investimentos/ } \\
\text { credenciamento de serviços }\end{array}$ & - Não se aplica \\
\hline & Atores & $\begin{array}{l}\text { - Institucionais: das três esferas de governo e instâncias colegiadas } \\
\text { do SUS (CIB, Conselho Estadual de Saúde, COSEMS, Conselho } \\
\text { de Gestão Regional) } \\
\text { - Prestadores privados } \\
\text { - Associações e organizações da sociedade civil }\end{array}$ & - Não se aplica \\
\hline & Estratégias & $\begin{array}{l}\text { - Criação ou fortalecimento de instâncias de pactuação e } \\
\text { coordenação regional } \\
\text { - Criação e revisão de instrumentos } \\
\text { - Reformas administrativas das Secretarias de Saúde } \\
\text { - Ampliação de recursos financeiros } \\
\text { - Ampliação de atores (públicos e privados, governamentais ou } \\
\text { não governamentais) }\end{array}$ & - Não se aplica \\
\hline & Instrumentos & $\begin{array}{l}\text { - Legislação, normas, incentivos, investimentos, planos, } \\
\text { mudanças de estruturas administrativas, contratualização, } \\
\text { processos participativos, capacitação, regulação da assistência, } \\
\text { consórcios }\end{array}$ & - Não se aplica \\
\hline
\end{tabular}




\begin{tabular}{|c|c|c|c|}
\hline \multicolumn{4}{|c|}{ Quadro 1. continuação } \\
\hline Dimensões & Categorias & Elementos principais & $\begin{array}{c}\text { Influência e padrões } \\
\text { predominantes }\end{array}$ \\
\hline \multirow[t]{2}{*}{$\begin{array}{l}\text { Características } \\
\text { da } \\
\text { regionalização }\end{array}$} & $\begin{array}{l}\text { Institucionalidade } \\
\text { da regionalização }\end{array}$ & $\begin{array}{l}\text { - Histórico da regionalização e conteúdo das regiões } \\
\text { (territorialidade) } \\
\text { - Definição/implantação de estratégias de planejamento e } \\
\text { regulação voltadas para coordenação de ações, serviços e } \\
\text { intervenções sanitárias em âmbito regional } \\
\text { - Definição/implantação de mecanismos de financiamento/ } \\
\text { investimentos voltados para a regionalização } \\
\text { - Papel da Secretaria de Estado de Saúde na condução da } \\
\text { regionalização: estruturas de coordenação definidas, } \\
\text { existência de estratégias políticas }\end{array}$ & $\begin{array}{l}\text { - Avançada } \\
\text { - Intermediária } \\
\text { - Incipiente }\end{array}$ \\
\hline & $\begin{array}{l}\text { Governança da } \\
\text { regionalização }\end{array}$ & $\begin{array}{l}\text { - Diversidade de atores e instâncias com peso na } \\
\text { regionalização } \\
\text { - Existência de mecanismos de coordenação das ações } \\
\text { conduzidas pelos atores com peso na regionalização } \\
\text { - Natureza das relações intergovernamentais e entre governos } \\
\text { e organizações } \\
\text { - Importância da CIB na regionalização }\end{array}$ & $\begin{array}{l}\text { - Coordenada/ } \\
\text { cooperativa } \\
\text { - Cooperativa } \\
\text { - Coordenada/ } \\
\text { conflitiva } \\
\text { - Conflitiva } \\
\text { - Indefinida }\end{array}$ \\
\hline
\end{tabular}

Fonte: Elaboração dos autores.

res a serem mobilizados, o objeto da regionalização e, finalmente, as estratégias e os instrumentos para auxiliar sua implementação.

Uma determinada institucionalidade da regionalização no plano estadual é conformada pela existência de recursos, incentivos, normas e construções cognitivas que integram o processo regulatório dessa política ${ }^{32}$. A noção de institucionalidade também está relacionada à trajetória da regionalização e aos elementos (conteúdo) levados em consideração no desenho das regiões de saúde no estado.

A governança da regionalização é determinada pela estabilidade do quadro institucional, pela abrangência dos atores, pelo tipo de relações estabelecidas entre governos e entre as organizações responsáveis pela condução do processo $^{33}$, sendo possível (ou não) estabelecer uma direção voltada para a consecução de objetivos e metas acordadas entre eles.

A pesquisa empírica, de natureza eminentemente qualitativa, privilegiou o período de 2007 a 2010. Esse período agrega a gestão estadual iniciada em 2007, incorporando o contexto de duas gestões municipais (2007 a 2008 e aquelas iniciadas em 2009) e de implantação do Pacto pela Saúde (vigente no período de 2007 a 2010).

Analisaram-se documentos, entre outros, atas e resoluções das Comissões Intergestores Bipartites estaduais, relatórios, planos estaduais (Pla- no Plurianual, Plano Estadual de Saúde, Plano Diretor de Regionalização, Plano Diretor de Investimentos, Programação Pactuada e Integrada), Leis e normativas propostas Secretarias de Estado de Saúde, atas dos Colegiados de Gestão Regional em funcionamento nos estados.

Também foram realizadas visitas por dupla de pesquisadores em 24 estados. Somente dois estados (Tocantins e Maranhão) não foram investigados, pois as equipes de gestão das Secretarias de Estado de Saúde tinham sido destituídas e não puderam ser contatadas no momento do trabalho de campo. No total, efetuaram-se 91 entrevistas semiestruturadas com 103 dirigentes e técnicos das Secretarias de Estado de Saúde e Conselhos de Secretários Municipais de Saúde em âmbito estadual (COSEMS): Secretários de Estado de Saúde, Presidentes dos COSEMS, responsáveis pela regionalização nos estados, Secretários Executivos das Comissões Intergestores Bipartites (CIB).

O cotejamento dessas diferentes fontes (documentos e entrevistas) à luz do referencial teórico, numa perspectiva crítico-reflexiva ${ }^{34}$, permitiu a identificação da influência e dos padrões preponderantes nas diversas dimensões analisadas (Quadro 1) e dos impactos gerados pela regionalização, em cada um dos estados estudados e seus agregados macrorregionais.

No que se refere à institucionalidade da regionalização, três estágios foram identificados: inci- 
piente, parcial e avançada. Já na categoria governança, um padrão coordenado/cooperativo se estabeleceu a partir de uma direção, com definição de metas e objetivos acordados e relações de cooperação entre os governos e as organizações responsáveis pela condução da regionalização. No mesmo sentido, a governança cooperativa foi estabelecida nos casos sem coordenação explícita entre as organizações, embora houvesse cooperação intergovernamental. Na governança coordenada/conflitiva, mesmo existindo um comando claro para o processo, muitos conflitos puderam ser observados sendo fracos os mecanismos de concertação. Por fim, o tipo conflitivo pressupôs um universo técnico-político conflituoso e com baixa coordenação entre os atores.

Os impactos institucionais gerados pela regionalização foram apreendidos pelas mudanças nas formas de funcionamento dos sistemas estaduais, na coordenação intergovernamental ou ainda nas estruturas e no papel da esfera estadual. Esses impactos foram classificados como radicais, incrementais, embrionários ou ausentes, levando-se em conta o ponto de partida, a intensidade e as velocidades das mudanças.

A pesquisa foi aprovada por Comitê de Ética da organização acadêmica que coordenou o estudo. Todos os entrevistados assinaram um Termo de Consentimento Livre e Esclarecido, cujo conteúdo assegurava-lhes o direito de recusa de participação e de sigilo das informações fornecidas.

\section{Resultados}

Na maior parte dos estados brasileiros do Norte e Nordeste, os contextos apresentavam-se desfavoráveis para o processo de regionalização, sendo que situação inversa foi observada nas demais macrorregiões do país (Quadro 2). Por outro lado, em nenhum dos estados investigados a conjuntura política foi avaliada como desfavorável, embora a situação se apresentasse com contornos mais indefinidos para a Região Norte.

Em dezenove estados o processo da regionalização orientou-se pela busca da equidade (Figura 1), sendo as desigualdades de financiamento e acesso aos serviços de saúde os principais obstáculos para a efetivação da universalização da saúde sugeridos pelos gestores entrevistados na pesquisa.

A busca de equidade associou-se à ideologia democrática-participativa em dezessete estados, e à ideologia gerencial em outros dezesseis, o que explica, respectivamente, a diversidade de atores e de interesses envolvidos nos processos decisórios e a ênfase em inovações na gestão do sistema.

Cinco estados orientaram-se pelo desenvolvimento integrado de políticas econômicas e sociais no âmbito estadual. O desenvolvimentismo, orientação que marca a gestão federal desde 2007, influenciou os estados governados pelo PT e pelo PMDB - partidos da base aliada do governo. Esses estados situavam-se nas regiões Norte (Amazonas e Pará) e Nordeste (Bahia, Ceará e Piauí).

Em quase a totalidade dos estados brasileiros observou-se que a organização de redes e fluxos, introduzida desde as primeiras normativas federais que versam sobre a regionalização do SUS, foi o objeto principal do processo de regionalização (Figura 1). Contudo, na maior parte dos estados (17 deles), a regionalização também teve como foco a ampliação da capacidade instalada, o que se explica pelo contexto de aumento da arrecadação e maior poder de investimento em saúde dos governos estaduais.

Ressalta-se a diversidade de atores envolvidos, com predomínio dos gestores e técnicos estaduais e municipais, participação de agentes privados (11 estados), universidades ( 3 estados), consórcios (3 estados) e representantes do Poder Legislativo (2 estados). Observou-se a importância da indução federal e da atuação do Ministério da Saúde, sobretudo, nos estados do Norte e Nordeste do país. A regionalização não apenas diversificou os atores, como contribuiu para o fortalecimento das instâncias de negociação intergovernamental (Comissões Intergestores Bipartites) e de representação dos secretários de saúde (Conselhos de Secretários Municipais de Saúde).

Destacaram-se como estratégias de indução da regionalização em saúde nos estados: a implantação e o fortalecimento de estruturas de coordenação federativa em âmbito regional (Colegiados de Gestão Regional e estruturas regionais das Secretarias de Estado de Saúde); a criação e a revisão de instrumentos de planejamento regional e regulação; e a ampliação dos investimentos na capacidade instalada e qualificação dos técnicos estaduais e municipais.

Foi possível observar a ênfase na programação de ações e recursos financeiros no processo de planejamento governamental. Contudo, a fragilidade da regulação da assistência à saúde apresentou-se como um problema em praticamente todos os estados.

Treze estados apresentaram institucionalidade intermediária do processo de regionalização. Já a governança apresentou-se como cooperati- 
Quadro 2. Contexto da regionalização em saúde nos estados segundo macrorregiões - Brasil, 2007 a 2010.

\begin{tabular}{|c|c|c|c|}
\hline Dimensões & $\begin{array}{l}\text { Histórico- } \\
\text { estrutural }\end{array}$ & $\begin{array}{c}\text { Político- } \\
\text { institucional }\end{array}$ & Conjuntural \\
\hline \multicolumn{4}{|l|}{ Estados } \\
\hline \multicolumn{4}{|c|}{ Região Norte } \\
\hline $\begin{array}{l}\text { Acre } \\
\text { Amapá } \\
\text { Amazonas } \\
\text { Pará } \\
\text { Rondônia } \\
\text { Roraima } \\
\text { Tocantins }\end{array}$ & $\begin{array}{c}\mathrm{D} \\
\mathrm{D} \\
\mathrm{D} \\
\mathrm{D} \\
\mathrm{D} \\
\mathrm{D} \\
\mathrm{NI}\end{array}$ & $\begin{array}{l}\mathrm{D} \\
\mathrm{D} \\
\mathrm{D} \\
\mathrm{D} \\
\mathrm{D} \\
\mathrm{D} \\
\mathrm{NI}\end{array}$ & $\begin{array}{c}\text { F } \\
\text { I } \\
\text { I } \\
\text { I } \\
\text { I } \\
\text { I } \\
\text { NI }\end{array}$ \\
\hline \multicolumn{4}{|c|}{ Região Nordeste } \\
\hline $\begin{array}{l}\text { Alagoas } \\
\text { Bahia } \\
\text { Ceará } \\
\text { Maranhão } \\
\text { Paraíba } \\
\text { Pernambuco } \\
\text { Piauí } \\
\text { Rio Grande do Norte } \\
\text { Sergipe }\end{array}$ & $\begin{array}{l}\mathrm{D} \\
\mathrm{D} \\
\mathrm{D} \\
\mathrm{NI} \\
\mathrm{D} \\
\mathrm{D} \\
\mathrm{D} \\
\mathrm{D} \\
\mathrm{F}\end{array}$ & $\begin{array}{c}\mathrm{D} \\
\mathrm{D} \\
\mathrm{F} \\
\mathrm{NI} \\
\mathrm{D} \\
\mathrm{D} \\
\mathrm{F} \\
\mathrm{D} \\
\mathrm{F}\end{array}$ & $\begin{array}{c}\text { I } \\
\text { F } \\
\text { F } \\
\text { NI } \\
\text { I } \\
\text { F } \\
\text { F } \\
\text { I } \\
\text { F }\end{array}$ \\
\hline \multicolumn{4}{|c|}{ Região Sudeste } \\
\hline $\begin{array}{l}\text { Espírito Santo } \\
\text { Minas Gerais } \\
\text { Rio de Janeiro } \\
\text { São Paulo }\end{array}$ & $\begin{array}{l}\mathrm{F} \\
\mathrm{F} \\
\mathrm{D} \\
\mathrm{F}\end{array}$ & $\begin{array}{l}\text { F } \\
\text { F } \\
D \\
\text { F }\end{array}$ & $\begin{array}{l}\mathrm{F} \\
\mathrm{F} \\
\mathrm{I} \\
\mathrm{F}\end{array}$ \\
\hline \multicolumn{4}{|c|}{ Região Sul } \\
\hline $\begin{array}{l}\text { Paraná } \\
\text { Rio Grande do Sul } \\
\text { Santa Catarina }\end{array}$ & $\begin{array}{l}\mathrm{F} \\
\mathrm{F} \\
\mathrm{F}\end{array}$ & $\begin{array}{l}\mathrm{F} \\
\mathrm{F} \\
\mathrm{D}\end{array}$ & $\begin{array}{l}\mathrm{F} \\
\mathrm{F} \\
\mathrm{F}\end{array}$ \\
\hline \multicolumn{4}{|c|}{ Região Centro-Oeste } \\
\hline $\begin{array}{l}\text { Goiás } \\
\text { Mato Grosso } \\
\text { Mato Grosso do Sul }\end{array}$ & $\begin{array}{l}\mathrm{F} \\
\mathrm{F} \\
\mathrm{F}\end{array}$ & $\begin{array}{l}\mathrm{D} \\
\mathrm{F} \\
\mathrm{F}\end{array}$ & $\begin{array}{l}\mathrm{I} \\
\mathrm{F} \\
\mathrm{F}\end{array}$ \\
\hline
\end{tabular}

Fonte: Entrevistas e documentos oficiais. Elaboração dos autores. Nota: F: favorável; D: desfavorável; I: indefinido; NI: Não investigado.

va ou coordenada-cooperativa para a maioria dos estados (14 estados). Identificou-se, ainda, a predominância de impactos incrementais associados à regionalização (Quadro 3).

A análise conjunta das categorias institucionalidade e governança sugeriu uma tipologia da regionalização em saúde nos estados brasileiros tendo como referencia dois tipos polares.

O primeiro evidencia uma maior maturidade do processo de regionalização (institucionalidade avançada) associado a um quadro institu- cional estável de integração entre os atores (governança coordenada/cooperativa). No outro extremo vê-se justamente o oposto, uma grande indefinição do papel dos atores e dos arranjos que possibilitam a articulação entre eles (governança conflitiva ou indefinida). Nesse caso, as estratégias desenvolvidas pela política de saúde mostraram-se frágeis e não conseguiram mobilizar atores estratégicos de forma a incrementar as capacidades técnicas, institucionais e políticas em prol da regionalização (institucionalidade in- 
cipiente). Entre esses dois extremos coexistem inúmeras combinações intermediárias (Figura 2).

\section{Discussão}

A análise da regionalização em saúde nos estados brasileiros no contexto do Pacto pela Saúde evidencia mudanças importantes no exercício de poder no interior da política de saúde, que se traduz por: introdução de novos atores (governamentais e não governamentais), objetos, regras e processos, orientados por diferentes concepções e ideologias; relevância das Secretarias de Estado de Saúde (SES) na condução da regionalização com fortalecimento das suas instâncias de representação regional; criação de novas instâncias de coordenação federativa (Colegiados de Gestão Regional); revisão das formas de organização e representatividade dos Conselhos de

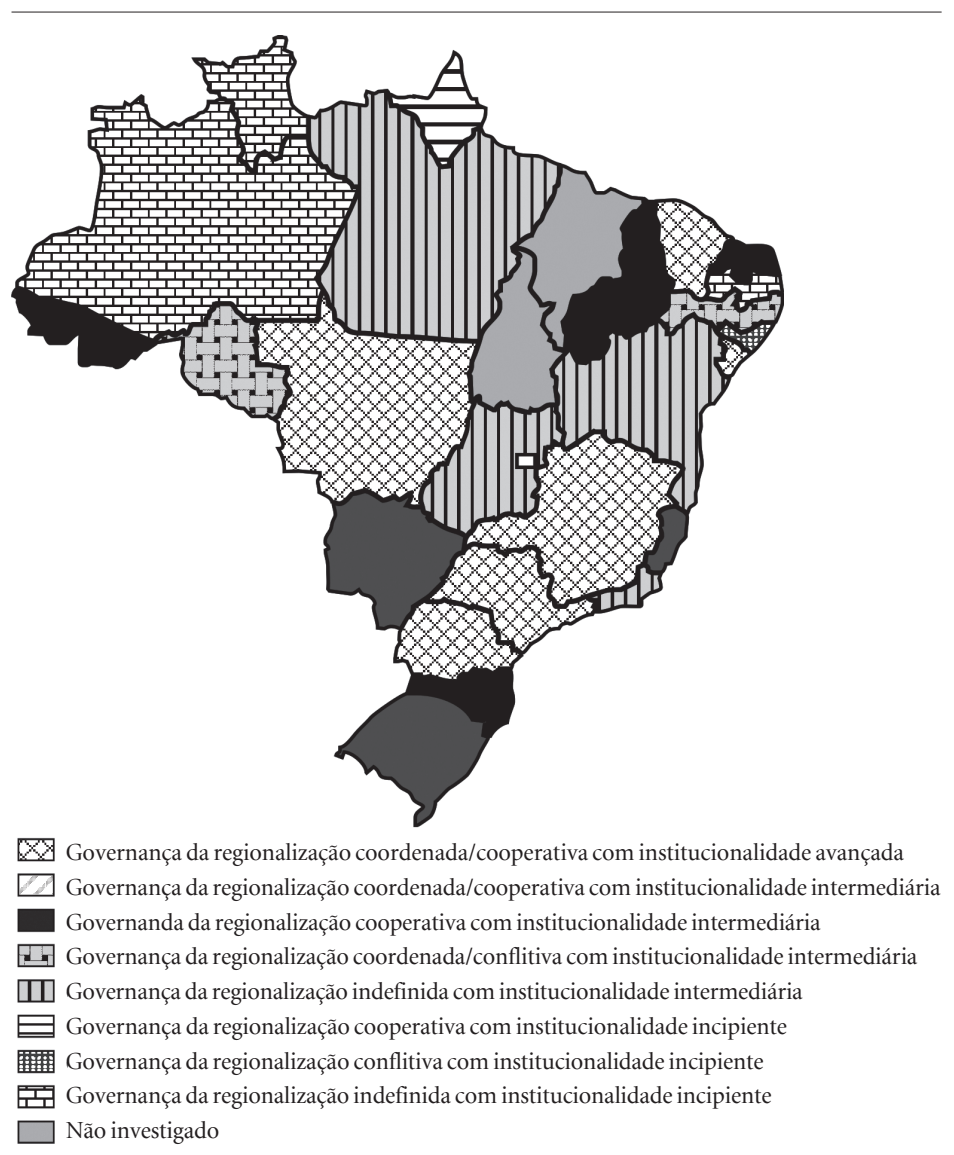

Figura 1. Ideologias e objetos predominantes no processo de regionalização da saúde nos estados - Brasil, 2007 a 2010.

Fonte: Entrevistas e documentos oficiais. Elaboração dos autores
Representação das Secretarias Municipais de Saúde e das Comissões Intergestores Bipartites; revisão dos acordos intergovernamentais estabelecidos na descentralização.

Nesse processo, enormes desafios se impõem para a governança e a atuação do Estado orientada pela lógica dos interesses coletivos e do SUS no espaço regional.

A análise também sugere a multiplicidade das experiências desenvolvidas no país. Isso porque, dado o importante papel adquirido pelas instâncias subnacionais (estados e municípios) na condução da regionalização, várias são as estratégias adotadas para lidar com a diversidade de contextos e lugares existentes. Além disso, diversas são as variáveis e a natureza dos fenômenos que condicionam e particularizam as experiências estaduais.

Assim, fatores de natureza histórico-estrutural, ligados à história de conformação dos estados, às dinâmicas socioeconômicas e às características dos sistemas de saúde são determinantes para o entendimento dos avanços e dificuldades enfrentadas. Também aspectos de ordem político-institucional, entre os quais se destacam o legado de implantação de políticas prévias (particularmente, de descentralização e regionalização), o aprendizado institucional acumulado pelas instâncias colegiadas e pelos governos estaduais e municipais (principalmente no que se refere às funções de planejamento e regulação), a existência de uma cultura de negociação intergovernamental, a qualificação técnica e política da burocracia governamental e os modos de operação e condução das políticas de saúde nos estados. Do mesmo modo, fatores conjunturais, particularmente aqueles relacionados à ação política, como o perfil e a trajetória dos atores políticos, a dinâmica das relações intergovernamentais e a prioridade da regionalização na agenda governamental, repercutem no processo decisório e nas escolhas realizadas.

Alguns estados brasileiros, por exemplo, foram conformados ainda no período colonial e apresentam processos muito antigos de regionalização na saúde, iniciados na primeira metade do século passado, incluindo a conformação de estruturas de representação regional das Secretarias de Saúde (tais como Minas Gerais, Rio Grande do Sul, São Paulo, Bahia e Pernambuco). Outros foram formados mais recentemente, sendo sua identidade estadual ou regional ainda muito incipiente. Neste caso, ressaltam-se: o Rio de Janeiro, que se constituiu em 1975 por meio da fusão entre o estado da Guanabara (antigo 
Quadro 3. Padrões predominantes do processo de regionalização em saúde nos estados segundo macrorregiões - Brasil, 2007 a 2010.

\begin{tabular}{|c|c|c|c|}
\hline Dimensões & Institucionalidade & Governança & $\begin{array}{c}\text { Impactos } \\
\text { institucionais }\end{array}$ \\
\hline \multicolumn{4}{|l|}{ Estados } \\
\hline \multicolumn{4}{|c|}{ Região Norte } \\
\hline $\begin{array}{c}\text { Acre } \\
\text { Amapá } \\
\text { Amazonas } \\
\text { Pará } \\
\text { Rondônia } \\
\text { Roraima } \\
\text { Tocantins }\end{array}$ & $\begin{array}{l}\text { Intermediária } \\
\text { Incipiente } \\
\text { Incipiente } \\
\text { Intermediária } \\
\text { Intermediária } \\
\text { Incipiente } \\
\text { NI } \\
\end{array}$ & $\begin{array}{c}\text { Cooperativa } \\
\text { Cooperativa } \\
\text { Indefinida } \\
\text { Indefinida } \\
\text { Coordenada-conflitiva } \\
\text { Indefinida } \\
\text { NI }\end{array}$ & $\begin{array}{c}\text { Incrementais } \\
\text { Embrionários } \\
\text { Ausentes } \\
\text { Embrionários } \\
\text { Embrionários } \\
\text { Ausentes } \\
\text { NI } \\
\end{array}$ \\
\hline \multicolumn{4}{|c|}{ Região Nordeste } \\
\hline $\begin{array}{c}\text { Alagoas } \\
\text { Bahia } \\
\text { Ceará } \\
\text { Maranhão } \\
\text { Paraíba } \\
\text { Pernambuco } \\
\text { Piauí } \\
\text { Rio Grande do Norte } \\
\text { Sergipe }\end{array}$ & $\begin{array}{c}\text { Incipiente } \\
\text { Intermediária } \\
\text { Avançada } \\
\text { NI } \\
\text { Incipiente } \\
\text { Intermediária } \\
\text { Intermediária } \\
\text { Intermediária } \\
\text { Avançada }\end{array}$ & $\begin{array}{c}\text { Conflitiva } \\
\text { Indefinida } \\
\text { Coordenada-cooperativa } \\
\text { NI } \\
\text { Indefinida } \\
\text { Coordenada-conflitiva } \\
\text { Cooperativa } \\
\text { Cooperativa } \\
\text { Coordenada-cooperativa }\end{array}$ & $\begin{array}{c}\text { Embrionários } \\
\text { Incrementais } \\
\text { Incrementais } \\
\text { NI } \\
\text { Embrionários } \\
\text { Incrementais } \\
\text { Radicais } \\
\text { Incrementais } \\
\text { Radicais }\end{array}$ \\
\hline \multicolumn{4}{|c|}{ Região Sudeste } \\
\hline $\begin{array}{l}\text { Espírito Santo } \\
\text { Minas Gerais } \\
\text { Rio de Janeiro } \\
\text { São Paulo }\end{array}$ & $\begin{array}{l}\text { Intermediária } \\
\text { Avançada } \\
\text { Intermediária } \\
\text { Avançada }\end{array}$ & $\begin{array}{c}\text { Coordenada-cooperativa } \\
\text { Coordenada-cooperativa } \\
\text { Indefinida } \\
\text { Coordenada-cooperativa }\end{array}$ & $\begin{array}{l}\text { Incrementais } \\
\text { Incrementais } \\
\text { Embrionários } \\
\text { Incrementais }\end{array}$ \\
\hline \multicolumn{4}{|c|}{ Região Sul } \\
\hline $\begin{array}{c}\text { Paraná } \\
\text { Rio Grande do Sul } \\
\text { Santa Catarina }\end{array}$ & $\begin{array}{c}\text { Avançada } \\
\text { Intermediária } \\
\text { Intermediária }\end{array}$ & $\begin{array}{c}\text { Coordenada-cooperativa } \\
\text { Coordenada-cooperativa } \\
\text { Cooperativa }\end{array}$ & $\begin{array}{l}\text { Incrementais } \\
\text { Incrementais } \\
\text { Incrementais }\end{array}$ \\
\hline \multicolumn{4}{|c|}{ Região Centro-Oeste } \\
\hline $\begin{array}{c}\text { Goiás } \\
\text { Mato Grosso } \\
\text { Mato Grosso do Sul }\end{array}$ & $\begin{array}{l}\text { Intermediária } \\
\text { Avançada } \\
\text { Intermediária }\end{array}$ & $\begin{array}{c}\text { Indefinida } \\
\text { Coordenada-cooperativa } \\
\text { Coordenada-cooperativa }\end{array}$ & $\begin{array}{l}\text { Incrementais } \\
\text { Incrementais } \\
\text { Incrementais }\end{array}$ \\
\hline
\end{tabular}

Fonte: Entrevistas e documentos oficiais. Elaboração dos autores. Nota: NI: Não investigado.

Distrito Federal) e o estado do Rio de Janeiro; o Tocantins; e os antigos territórios brasileiros transformados em estados (Amapá, Roraima e Rondônia).

Além disso, também podem atuar como elementos decisivos na regionalização, a experiência acumulada no planejamento governamental, as formas de organização e cultura de negociação intergovernamental adquiridas pelas secretarias estaduais e municipais de saúde e instânci- as colegiadas no estado, a experiência com estratégias de formalização de parcerias (por exemplo, consórcios de saúde e contratos de gestão). Nessa situação, encontram-se os estados do Ceará, Mato Grosso, Mato Grosso do Sul, Minas Gerais, Paraná e São Paulo. Cabe destacar que, em alguns estados, a regionalização foi potencializada pela articulação de políticas governamentais que ampliaram o alcance das proposições do setor saúde. 


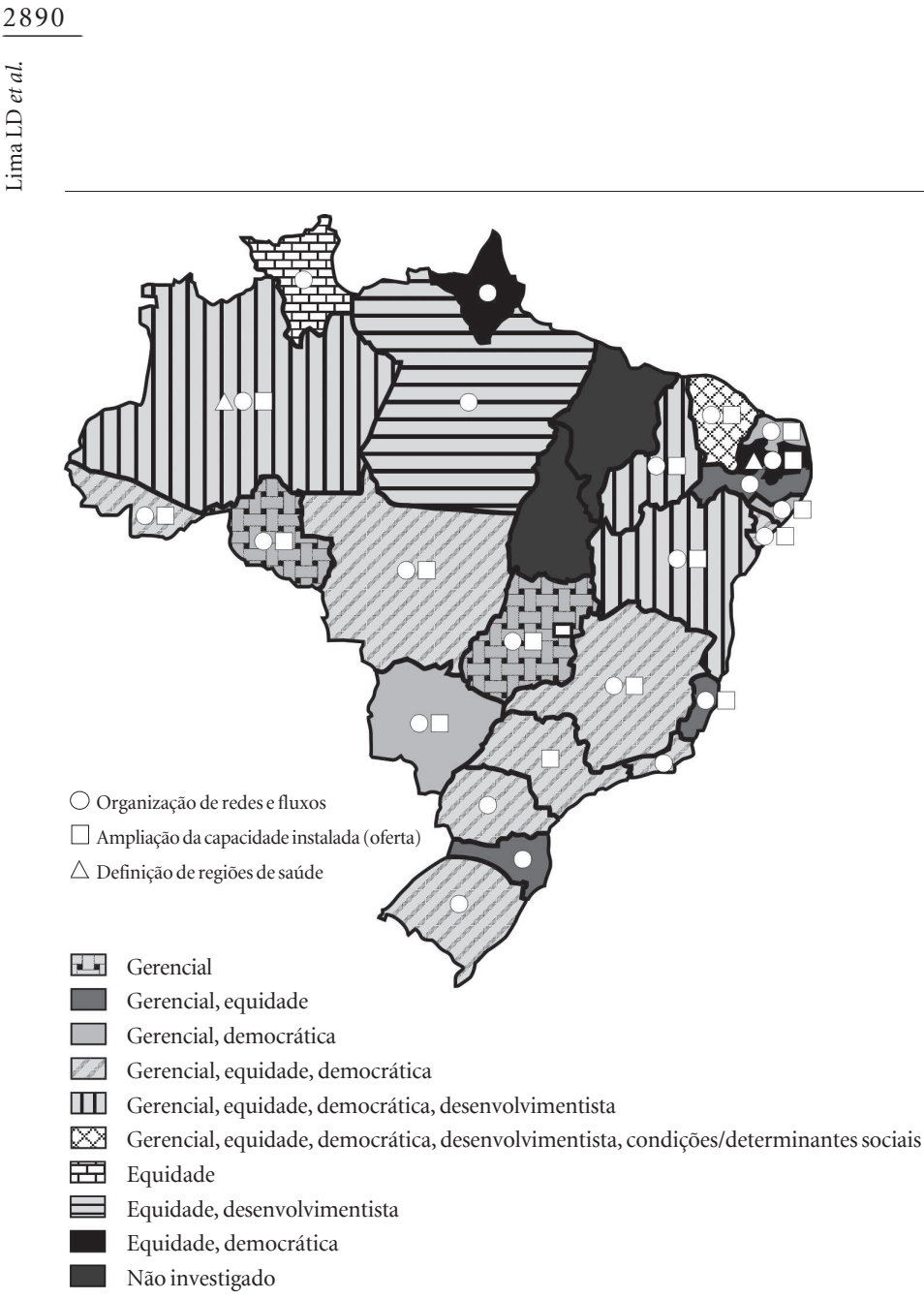

Figura 2. Tipologia do processo de regionalização da saúde nos estados - Brasil, 2007 a 2010.

Fonte: Entrevistas e documentos oficiais. Elaboração dos autores.

Algumas dificuldades para atuação regional nos estados decorrem do fato de que, muitas vezes, a integração dos serviços de saúde obedece a lógicas territoriais da rede urbana que extrapolam suas fronteiras (municípios cujos territórios se relacionam com outros estados). Há também forte ingerência do poder político eleitoral (os chamados bolsões eleitorais de determinados políticos) em certas regiões, e pesadas heranças centralizadoras em alguns estados da federação (como refletem as experiências dos estados do Nordeste). As longas distâncias que separam as sedes municipais e a precariedade das redes de transporte e comunicação, sobretudo na região Norte, dificultam a assiduidade dos gestores municipais nos fóruns de pactuação intergovernamentais, incluindo os Colegiados de Gestão Regional.
Outras razões vinculam-se às desigualdades inter e intrarregionais, marcadas pela alta concentração de recursos e tecnologias em algumas regiões (principalmente, áreas metropolitanas ou sede de capitais em sua maioria situadas no litoral), em oposição à escassez de profissionais, tecnologias e capacidade de investimento somada à diversidade socioespacial de alguns territórios (caso dos estados da região Norte).

Além disso, a atuação de interlocutores com forte representatividade político-institucional, na negociação e mediação de conflitos, tem se constituído como fator distintivo entre os estados nos processos de regionalização. Essa atuação pode ser identificada tanto entre os gestores municipais, quando agem de forma organizada e participativa, quanto no âmbito das secretarias estaduais de saúde, pela sua capacidade de diálogo e coordenação dos processos.

A presença de atores públicos com legitimidade e poder político necessário para a superação de conflitos - comuns em momentos de renovação de práticas gestoras - quando associados, no âmbito institucional, à existência de equipes técnicas qualificadas, tem sido decisiva para a adoção de novas experiências de planejamento e gestão em saúde (como sugere a experiência de Sergipe).

Em síntese, a regionalização se associa, em cada estado, às dinâmicas territoriais, às características do desenvolvimento econômico, às políticas de saúde anteriores, ao grau de articulação existente entre os representantes do Conselho de Representação das Secretarias Municipais de Saúde e da Secretaria Estadual de Saúde, no sentido de gerar consensos sobre a divisão de responsabilidades gestoras e os desenhos regionais adotados em cada estado.

Os resultados sugerem ganhos de institucionalidade em contextos histórico-estruturais e políticos-institucionais desfavoráveis. Entretanto, as dificuldades inerentes a esses contextos comprometem maiores avanços e a sustentabilidade da política de regionalização em muitas regiões. Percebe-se, portanto, a necessidade de fortalecimento do enfoque territorial no planejamento governamental e da integração de políticas setoriais a de desenvolvimento regional de médio e longo prazo. Essa é uma condição fundamental para ampliar e assegurar o acesso da população aos serviços de saúde de diversos tipos, conforme as diretrizes do SUS de universalidade e integralidade. 


\section{Colaboradores}

LD Lima, ALD Viana, CV Machado e MV de Albuquerque foram responsáveis pela concepção, coleta e análise das informações e redação do artigo. RG de Oliveira, FL Iozzi, JHG Scatena, GA Mello, AMM Pereira e APS Coelho participaram da coleta e análise das informações, confecção dos quadros e figuras e revisão final do artigo.

\section{Agradecimentos}

LD Lima, ALD Viana e CV Machado são bolsistas de produtividade do Conselho Nacional de Desenvolvimento Científico e Tecnológico (CNPq). A pesquisa contou com recursos do Ministério da Saúde, em parceria com a Organização Panamericana de Saúde, do CNPq e da Fundação Carlos Chagas de Amparo à Pesquisa do Estado do Rio de Janeiro (FAPERJ). Agradecemos às equipes do Departamento de Apoio a Gestão Descentralizada do Ministério da Saúde, da Secretaria Técnica da Comissão Intergestores Tripartite, das Secretarias de Estado de Saúde, dos Conselhos de Secretarias Municipais de Saúde no âmbito dos es tados e das Comissões Intergestores Bipartites, pelo apoio recebido no desenvolvimento dessa pesquisa.

\section{Referências}

1. Melo AC. Crise federativa, guerra fiscal e "hobbesianismo municipal": efeitos perversos da descentralização? São Paulo Perspec 1996; 10(3):11-20.

2. Bankauskaite V, Dubois HFW, Saltman RB. Patterns of decentralization across European health systems. In: Saltaman RB, Bankauskaite V, Vrangbaek K, organizadores. Decentralization in health care: strategies and outcomes. Maidenhead: Open University Press; 2007. p. 22-43.

3. Organización Panamericana de la Salud (OPAS). Redes Integradas de Servicios de Salud: Conceptos, Opciones de Política y Hoja de Ruta para su Implementación en las Américas. Washington DC: OPAS; 2010.

4. Ribeiro PT. A descentralização da ação governamental no Brasil dos anos noventa: desafios do ambiente político-institucional. Cien Saude Colet 2009; 13(3):819-828.

5. Viana ALD, Lima LD, Oliveira RG. Descentralização e federalismo: a política de saúde em novo contexto - lições do caso brasileiro. Cien Saude Colet 2002; 7(3):493-507.

6. Melo AC. O sucesso inesperado das reformas de segunda geração: federalismo, reformas constitucionais e política social. Dados 2005; 48(4):845-889.

7. Almeida MHT. O Estado no Brasil contemporâneo. In: Melo CR, Sáez MA, organizadores. A Democracia Brasileira: balanço e perspectivas para o século 21. Belo Horizonte: Ed. UFMG; 2007. p. 17-37.

8. Arretche MTS. Continuidades e descontinuidades da Federação Brasileira: de como 1988 facilitou 1995. Dados 2009; 52(2):377-423.

9. Viana ALD, Fausto MCR, Lima LD. Política de saúde e equidade. São Paulo Perspec 2003; 17(1):58-68.

10. Brasil. Portaria GM/MS n. 95 , de 26 jan. 2001. Aprova a norma operacional da assistência à saúde - NOASSUS 01/01. Diário Oficial da União 2001; 26 jan.

11. Brasil. Ministério da Saúde (MS). Regionalização da Assistência à Saúde: aprofundando a descentralização com equidade no acesso. Brasília: MS; 2002.

12. Brasil. Portaria GM/MS n.399, de 22 de fevereiro de 2006. Diário Oficial da União 2006; 23 fev.

13. Brasil. Portaria GM/MS n.699, de 30 de março de 2006. Diário Oficial da União 2006; 03 abr.

14. Viana ALD, Ibañez N, Elias PEM, Lima LD, Albuquerque M, Iozzi FL. Novas perspectivas para a regionalização da saúde. São Paulo Perspec 2008; 22(1):92-106.

15. Arretche MTS. Federalismo e relações intergovernamentais no Brasil: a reforma de programas sociais. Dados 2002; 45(3):431-458.

16. Machado CV. O modelo de intervenção do Ministério da Saúde nos anos 90. Cad Saude Publica 2007; 23(9):2113-2126.

17. Lima LD, Machado CV, Baptista TWF, Pereira AMM. O Pacto Federativo brasileiro e o papel do gestor estadual no SUS. In: Ugá MA, Sá MC, Martins $\mathrm{M}$, Braga Neto F, organizadores. A gestão do SUS no âmbito estadual: o caso do Rio de Janeiro. Rio de Janeiro: Fiocruz; 2010. p. 27-58.

18. Souza C. Governos e sociedades locais em contextos de desigualdades e de descentralização. Cien Saude Colet 2002; 7(3):431-442. 
19. Campos GWS. Efeitos paradoxais da descentralização do Sistema Único de Saúde do Brasil. In: Fleury S, organizadora. Democracia, descentralização e desenvolvimento: Brasil e Espanha. Rio de Janeiro: FGV; 2006. p. 417-442.

20. Brasil. Ministério da Saúde (MS). Regionalização solidária e cooperativa: orientações para sua implementação no SUS. Brasília: MS; 2006. (Série Pactos pela Saúde, v. 3)

21. Brasil. Ministério da Saúde (MS). Colegiado de Gestão Regional na região de saúde intra-estadual: orientações para organização e funcionamento (Série Pactos pela Saúde, v.10). Brasília: MS; 2009.

22. Viana ALD, Lima LD, organizadores. Regionalização e relações federativas na política de saúde do Brasil. Rio de Janeiro: Contra Capa; 2011.

23. Souza RR. A regionalização no contexto atual das políticas de saúde. Cien Saude Colet 2001; 6(2): 451455.

24. Mendes EV. As redes de atenção à saúde. Cien Saude Colet 2010; 15(5):2297-2305.

25. Kuschnir R, Chorny AH. Redes de atenção à saúde: contextualizando o debate. Cien Saude Colet 2010; 15(5):2307-2316

26. Gadelha CAG, Machado CV, Lima LD, Baptista TWF. Saúde e territorialização na perspectiva do desenvolvimento. Cien Saude Colet 2011; 16(6):3003-3016.

27. Ham C, Hill M. The Policy Process in the Modern Capitalist State. Hertfordshire: Haverster Wheatsheaf; 1993.

28. Thelen K, Steinmo S, editors. Structuring Politics. Historical Institucionalism in Comparative Analysis. Cambridge: Cambridge University Press; 1992.

29. Pierson P. Politics in time: history, institutions, and social analysis. New Jersey: Princeton University Press; 2004.

30. Hall P, Taylor RCR. As três versões do neo-institucionalismo. Lua Nova 2003; 58:193-223.

31. Denis J-L. Reforme et gouvernance en santé: l'attrait pour un managérialization de l'action publique. In : Rocher G, Noreau P, Laborier P, Rioux M, organizadores. Reforme en santé et en justice: le droit et la gouvernance. Montréal: Les Presses de L’Université Laval; 2008.

32. Scott RW. Institutions and Organizations. Thousand Oaks: Sage; 1995.

33. Defarges PM. La Gouvernance. Paris: PUF; 2008.

34. Minayo MCS. O desafio do conhecimento: pesquisa qualitativa em saúde. São Paulo: Editora Hucitec; 2006. 